\title{
The migration of synthetic magnetic nanoparticle labeled dendritic cells into lymph nodes with optical imaging
}

This article was published in the following Dove Press journal:

International Journal of Nanomedicine

I October 2013

Number of times this article has been viewed

\author{
Hang Su, ${ }^{1, *}$ \\ Yongbin Mou ${ }^{1, *}$ \\ Yanli $A n^{2}$ \\ Wei Han' \\ Xiaofeng Huang' \\ Guohua $\mathrm{Xia}^{3}$ \\ Yanhong $\mathrm{Ni}^{1}$ \\ Yu Zhang ${ }^{4}$ \\ Jianmin $\mathrm{Ma}^{\prime}$ \\ Qingang $\mathrm{Hu}^{1,5}$ \\ 'Center Laboratory of Stomatology, \\ Stomatological Hospital Affiliated Medical \\ School, Nanjing University, Nanjing, \\ People's Republic of China; ${ }^{2}$ Jiangsu Key \\ Lab of Molecular and Function Imaging, \\ Department of Radiology; ${ }^{3}$ Department \\ of Hematology, Zhongda Hospital, Medica \\ School, ${ }^{4}$ State Key Laboratory of Molecule \\ and Bimolecular Electronics, Jiangsu \\ Provincial Laboratory for Biomaterials and \\ Devices; Southeast University, Nanjing, \\ People's Republic of China; ${ }^{5}$ Leeds Dental \\ Institute, Faculty of Medicine and health, \\ University of Leeds, Leeds, United Kingdom \\ *These authors contributed equally to this \\ work
}

Correspondence: Qingang Hu

Center Laboratory of Stomatology,

Stomatological Hospital Affiliated Medical

School, Nanjing University, 30 Zhongyang

Road, Nanjing 210008, People's Republic

of China

$\mathrm{Tel}+86258362$ 0l0l

Fax +86258362 0101

Email qghu@nju.edu.cn

Yongbin Mou

Center Laboratory of Stomatology,

Stomatological Hospital Affiliated Medical

School, Nanjing University, 30 Zhongyang

Road, Nanjing 210008 , People's Republic

of China

Tel +86 2583620236

Fax +86258362 010।

Email yongbinmou@gmail.com
Background: The successful biotherapy of carcinoma with dendritic cell (DC) vaccines pivotally relies on DCs' migratory capability into lymph tissues and activation of T cells. Accurate imaging and evaluation of DC migration in vivo have great significance during antitumor treatment with DC vaccine. We herein examined the behavior of DCs influenced by synthetic superparamagnetic iron oxide (SPIO) nanoparticle labeling.

Methods: $\gamma-\mathrm{Fe}_{2} \mathrm{O}_{3}$ nanoparticles were prepared and DCs, which were induced from bone marrow monocytes of enhanced green fluorescent protein (EGFP) transgenic mice, were labeled. The endocytosis of the SPIO, surface molecules, cell apoptosis and fluorescence intensity of EGFP-DCs were displayed by Prussian blue staining and flow cytometry (FCM), respectively. After EGFP-DCs, labeled with SPIO, were injected into footpads $(n=5)$ for 24 hours, the mice were examined in vivo by optical imaging (OPI). Meanwhile, confocal imaging and FCM were applied, respectively, to detect the migration of labeled DCs into draining lymph nodes.

Results: Nearly $100 \%$ of cells were labeled by the SPIO, in which the intracellular blue color gradually deepened and the iron contents rose with the increase of labeling iron concentrations. In addition, cell apoptosis and the surface molecules on DCs were at similar levels after SPIO labeling. After confirming that the fluorescence intensity of EGFP on DCs was not influenced by SPIO, the homing ability of EGFP-DCs labeled with SPIO displayed that the fluorescence intensity and the ratios of EGFP-DCs in draining lymph nodes were gradually decreased with the increase of labeling iron concentrations.

Conclusion: The synthetic SPIO nanoparticles possess perfect labeling ability and biocompatibility. Moreover, DCs labeled with a low dose of SPIO showed stronger migratory capability in vivo.

Keywords: optical imaging, dendritic cell, superparamagnetic iron oxide, cell tracking

\section{Introduction}

Due to the multifunctional properties for biomedical applications, superparamagnetic iron oxide (SPIO) nanoparticles have been regarded as a useful tool in numerous medical diagnoses or treatment, ${ }^{1-3}$ the applications of which includes in vivo cell tracking, hyperthermia therapy, lymph node detection, and anticancer drug delivery, etc. ${ }^{4-7}$ In particular, SPIO nanoparticles can enhance contrast in magnetic resonance imaging (MRI), which allows researchers to monitor anatomical, physiological, and molecular changes during the evolution of a disease or treatment.

Dendritic cells (DCs) are professional antigen-presenting cells that play a critical role in the initiation of primary immune responses and stimulate naïve T cells. ${ }^{8}$ Accumulated data have shown that DCs can induce powerful antitumor immune 
responses by using their exceptional capabilities to capture antigens, process and present antigenic peptide fragments, migrate into lymphoid organs, and strengthen primary immune responses. ${ }^{9}$ DC vaccines loaded with the tumor antigen and then transfused into patients with cancer is currently one of the strategies with the most potential, which has the advantages of high efficiency, low toxicity, low side effects, and so on. Although it is difficult to prove the immunomonitoring of cellular immunotherapies, we have recently demonstrated that mouse bone marrow DCs could be safely labeled with our synthetic SPIO nanoparticles, ${ }^{10}$ meanwhile, the labeled DCs migrating into the draining popliteal lymph nodes could be monitored by the hypersensitive 7T MRI and optical imaging (OPI) techniques effectively through footpad injections. ${ }^{11}$ With the help of the MRI and OPI, we could assess the delivery of enhanced green fluorescent protein (EGFP) transgenic DCs labeled with SPIO into the lymph nodes accurately. However, only a few of the labeled DCs migrating into draining lymph nodes were observed while most cells stayed at the injected section and underwent cell apoptosis in our and other previous studies. ${ }^{12}$

As accurate delivery and targeting are crucial for the efficacy of cellular therapies, it is urgent to demonstrate imaging of cellular vaccines precisely after injection into the patient and the improvement of the efficacy of the antitumor immunity. From the data presented here, we conclude that SPIO labeled EGFP transgenic DCs migrating into local draining lymph nodes can be detected when the iron concentration for labeling is $25 \mu \mathrm{g} / \mathrm{mL}$, with only about $4 \%$ labeled DCs migrating into local draining lymph nodes. But how would labeling DCs with different concentrations of synthetic SPIO influence this process?

The purpose of this study is to evaluate the impact of synthetic SPIO on DCs and to explore the migratory efficiency of DCs labeled with different concentrations of SPIO using OPI techniques.

\section{Materials and methods Mice}

Female C57BL/6 mice and EGFP-transgenic mice of C57BL/6 background were purchased from Model Animal Research Center of Nanjing University (Nanjing, People's Republic of China) and were kept under specified pathogenfree conditions in the Central Animal Facility, Nanjing University. For experimental purposes, the bone marrow donors were between 8 to 10 weeks old and the weight of the donors ranged from 20 to $22 \mathrm{~g}$. All mice involved in the experiments were performed in accordance with protocols approved by the Animal Care and Use Committee of the Medical School, Nanjing University.

\section{Cell culture}

Monocytes, which were isolated from bone marrow of mice, were cultured with Roswell Park Memorial Institute (RPMI) medium 1640 with $100 \mathrm{U} / \mathrm{mL}$ penicillin, $100 \mathrm{mg} / \mathrm{mL}$ streptomycin (Gibco-Invitrogen, Grand Island, NY, USA), 10\% fetal bovine serum (Gibco, Life Technologies, Breda, The Netherlands), and $10 \mathrm{ng} / \mathrm{mL}$ granulocyte-macrophage colonystimulating factor and $1 \mathrm{ng} / \mathrm{mL}$ interleukin (IL)-4 (eBioscience, San Diego, CA, USA). On days 2 and 4, half of the medium was removed and fresh medium was renewed. The nonadherent cells were washed out on day 6 and were incubated with tumor necrosis factor (TNF- $\alpha ; 10 \mathrm{ng} / \mathrm{mL}), \mathrm{IL}-1 \beta(10 \mathrm{ng} / \mathrm{mL})$, IL-6 (10 ng/mL; Peprotech, Rocky Hills, NJ, USA) and prostaglandin $\mathrm{E}_{2}(1 \mu \mathrm{g} / \mathrm{mL}$; Sigma-Aldrich, St Louis, MO, USA) for 12 hours. ${ }^{13}$ The mature DCs were collected on day 7 .

\section{Cell labeling}

The collected DCs were seeded in a 6-well plate and incubated at $37^{\circ} \mathrm{C}$ with $5 \% \mathrm{CO}_{2}$. SPIO nanoparticles were prepared according to our previous work. ${ }^{14}$ Different concentrations of SPIO $(0,10,25,50 \mu \mathrm{g} / \mathrm{mL})$ were added to the cell medium. After incubation of 12 hours, the medium was removed, the cells were washed and cytospins were prepared. Thereafter, the amount of intracellular iron was visualized using a Prussian blue staining kit (Shanghai Yuanye BioTechnology Co, Ltd, People's Republic of China) by which, according to the supplier's instructions, the iron reacts with acid ferrocyanide to produce a blue color. Screenings were observed with a light microscope (Olympus, CKX41-A32PH, Tokyo, Japan).

\section{Flow cytometry assay}

For the phenotypic characterization assay, surface markers were tested by FCM (flow cytometry) (FACSCalibur; BD Biosciences). The following monoclonal antibodies were used for the immunophenotyping of DCs: fluorescein isothiocyanate (FITC)-MHC-II, phycoerythrin (PE)-CD80, PE-CD86, and PE-CCR7 (eBioscience) according to the manufacturer's instructions. Meanwhile, cell apoptosis was also assayed with an Annexin V-FITC and Propidium iodide (PI) apoptosis kit, according to the recommendations of the manufacturer (Biouniquer, Nanjing, People's Republic of China). After DCs were gated, ten thousand gated cells 
were analyzed per sample and the detectors for FITC, PE, PI, were FL1, FL2, FL3 respectively.

\section{Quantification of internal iron}

DCs were seeded $\left(5 \times 10^{5}\right)$ and washed twice with phosphate buffered saline (PBS) after they were treated with SPIO $(0,10,25,50 \mu \mathrm{g} / \mathrm{mL})$ for 12 hours. The amount of iron inside the cells was investigated according to the Ge method. ${ }^{15}$ A standard curve (not shown) of an aqueous $\mathrm{FeCl}_{3} \cdot 6 \mathrm{H}_{2} \mathrm{O}$ solution was adopted under the same conditions to quantify the amount of the SPIO in cellular uptake. Also, the iron content of the SPIO-labeled DCs was expressed as the amount of $\mathrm{Fe}_{2} \mathrm{O}_{3}$.

\section{OPI ex vivo}

After incubation with SPIO $(0,10,25,50 \mu \mathrm{g} / \mathrm{mL})$ for 12 hours and washed thrice, EGFP fluorescence intensity of cells was studied by FCM with Cell Quest software (BD Biosciences). Then, for in vivo imaging, an inflammatory factor (TNF- $\alpha 30 \mathrm{ng} / \mathrm{side})^{11}$ was preinjected into the hind footpads of C57BL/6 mice $(\mathrm{n}=5)$. After 24 hours, $2 \times 10^{6}$ EGFP-DCs loaded with various concentrations of SPIO were injected into the footpads and the control group was injected with EGFP-DCs without SPIO.

To detect the migration of DCs into draining lymph nodes ex vivo, the optical imaging of dissected popliteal and inguinal lymph nodes was performed after the administration of DCs for 24 hours using the Maestro in vivo imaging system (CRi, Woburn, MA, USA). The $484 \mathrm{~nm}$ excitation and emission filters $(507 \mathrm{~nm})$ were applied to observe the dissected lymph nodes. The fluorescent images consisting of EGFP-related fluorescence were then organized based on their spectral patterns with Living Image software (v 2.50; Caliper Corporation, Newton, MA, USA).

\section{Immunohistochemistry}

For confocal microscopic analyses, frozen lymph nodes were cryosectioned into $5 \mu \mathrm{m}$-thick sections and fixed with ice-cold acetone. The sections were incubated with a rabbit anti-mouse GFP antibody (Invitrogen) and washed with PBS and goat anti-rabbit Alexa $488 \mathrm{~nm}$ antibody (Invitrogen) was used as the secondary antibody. Samples were observed with a confocal laser scanning microscope (Fluoview, Fv10i; Olympus).

\section{Percentage of EGFP-DCs}

For comparing the percentages of DCs in draining lymph nodes, $2 \times 10^{6}$ EGFP-DCs labeled with SPIO were infused into the footpads of mice. After 24 hours, the lymph nodes were anatomized and pooled. Next, a single cell suspension was prepared with PBS and the fluorescence of EGFP was examined by FCM. During examination, $5 \times 10^{6}$ cells were analyzed per sample and the detector for EGFP was FL1.

\section{Statistical analysis}

Results are expressed as means \pm standard deviation (SD). The statistical significance of the differences between the groups was assessed by one-way analysis of variance (ANOVA) and two-tailed Student's $t$-test. A $P$-value $<0.05$ was considered statistically significant.

\section{Results \\ Efficiency and cellular uptake of SPIO labeling}

To evaluate the potential for SPIO particles in tracking DCs, SPIO particles with different concentrations phagocytized by DCs were examined first. Prussian blue staining showed that almost all cells were labeled by SPIO and the intracellular blue color gradually deepened with increasing iron concentrations (Figure 1).

SPIO particles internalized by DCs were also analyzed. On average, the iron contents in SPIO-DCs were $14.09 \pm$ $0.25 \mu \mathrm{g}, 20.12 \pm 1.28 \mu \mathrm{g}$, and $25.00 \pm 1.69 \mu \mathrm{g}$ per $1 \times 10^{6}$ cells, respectively, according to the labeling concentration of SPIO nanoparticles (Figure 2).
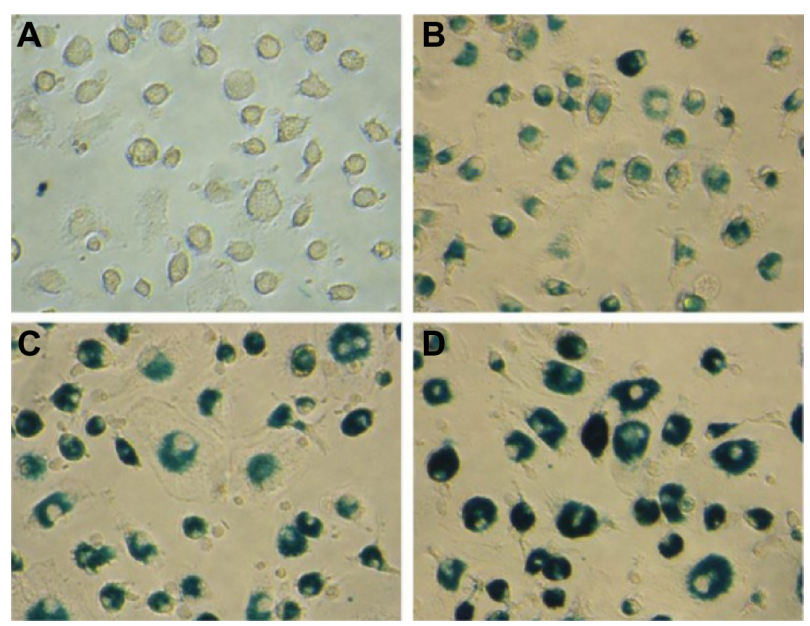

Figure I Morphology of DCs labeled with SPIO $(0,10,25,50 \mu \mathrm{g} / \mathrm{mL})$ after 24 hour incubation with Prussian blue staining $(\times 400)$.

Notes: (A) DCs; (B) DCs (SPIO, $10 \mu \mathrm{g} / \mathrm{mL}$ ); (C) DCs (SPIO, $25 \mu \mathrm{g} / \mathrm{mL}$ ); (D) DCs (SPIO, $50 \mu \mathrm{g} / \mathrm{mL}$ ).

Abbreviations: DCs, dendritic cells; SPIO, superparamagnetic iron oxide. 


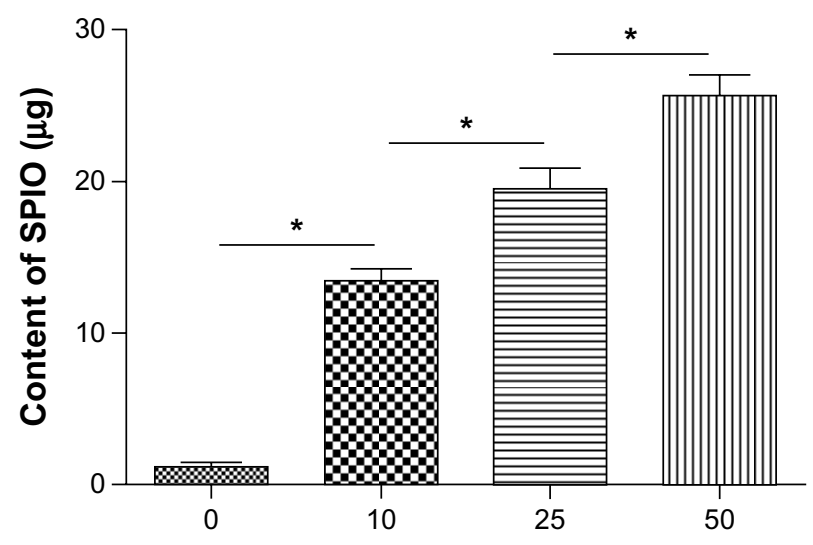

Labeling concentration of SPIO $(\mu \mathrm{g} / \mathrm{mL})$

Figure 2 Cellular uptake of iron by DCs at 24 hours co-culture time.

Notes: Mean concentrations of iron in DCs (per I $\times 10^{6}$ cells) after co-culturing with $0,25,25,50 \mu \mathrm{g} / \mathrm{mL}$ SPIO for 24 hours. $* P=0.0156$

Abbreviations: DCs, dendritic cells; SPIO, superparamagnetic iron oxide.

\section{Cell phenotypes}

The surface molecules of mature DCs labeled with different concentrations of SPIO were characterized by FCM. The expression of CD80, CD86, MHC-II, and CCR7 of DCs did not have a detectable increase compared to those without SPIO labeling (Figure 3).

\section{Cell apoptosis assay}

To test whether the apoptosis of DCs labeled with the different concentrations of SPIO nanoparticles was influenced, DCs and SPIO-DCs were analyzed by FCM after Annexin V and PI staining; there were no significant differences in the total percentage of Annexin V/PI DCs at all concentration points (Figure 4).

\section{EGFP fluorescence intensity after SPIO labeling}

To analyze EGFP fluorescence of DCs, FCM was performed and the results revealed no significant differences in EGFP fluorescence intensity among the groups regardless of SPIO labeling concentrations (Figure 5).

\section{OPI ex vivo}

To detect the amount of DCs migrating into lymph nodes, the draining lymph nodes were dissected and examined by OPI ex vivo. The images with different brightness confirmed the migration of SPIO labeled EGFP-DC into the popliteal lymph nodes. With the increasing iron concentrations, the fluorescence intensity of EGFP in popliteal lymph nodes decreased gradually and no signals were displayed in the
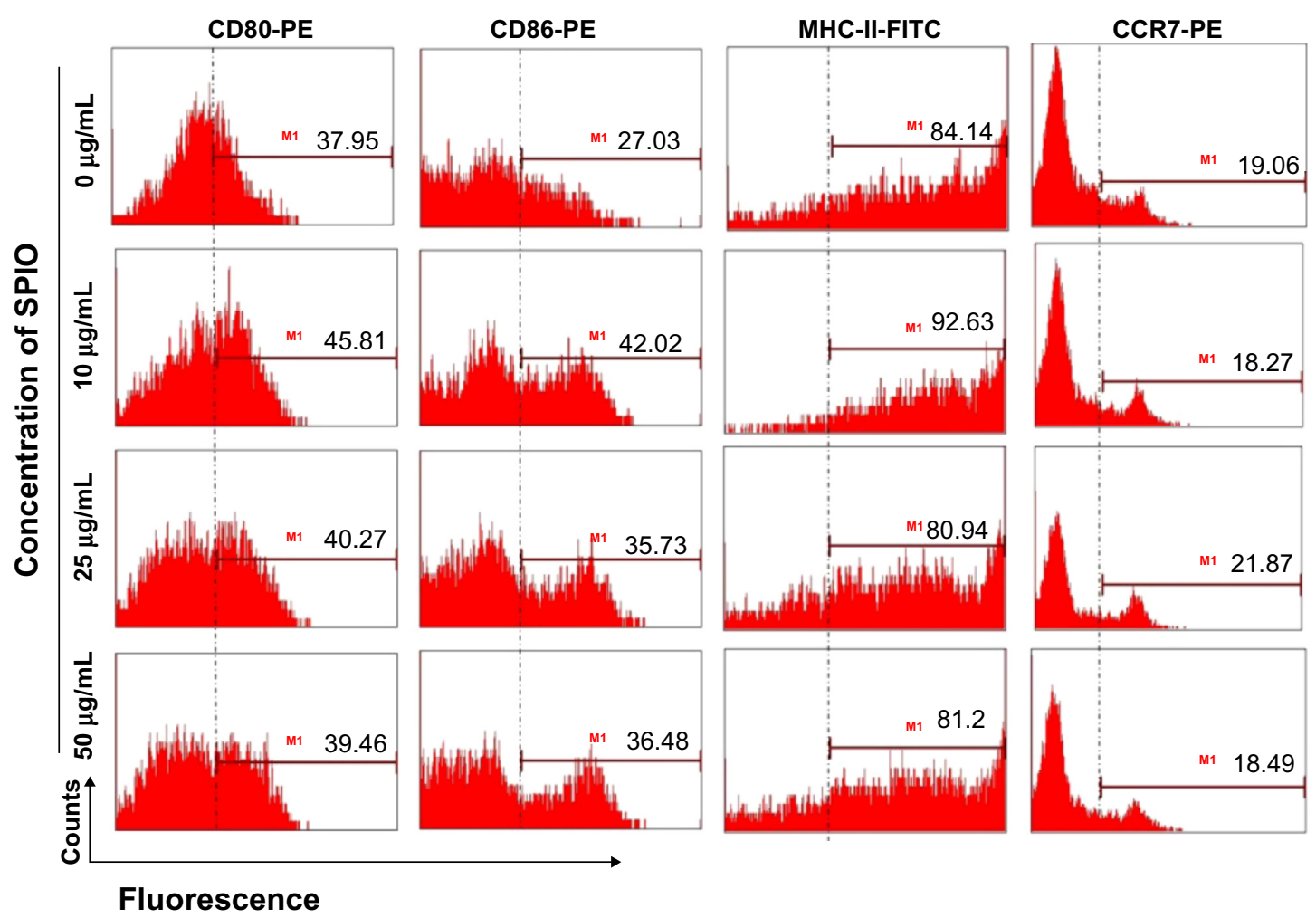

Fluorescence

Figure 3 Phenotypes of DCs after labeling with SPIO $(0,10,25,50 \mu \mathrm{g} / \mathrm{mL} ; P>0.05)$.

Abbreviations: CCR, chemokine receptor; CD, cluster of differentiation; DCs, dendritic cells; FITC, fluorescein isothiocyanate; MHC, major histocompatibility complex; $\mathrm{PE}$, phycoerythrin; SPIO, superparamagnetic iron oxide. 


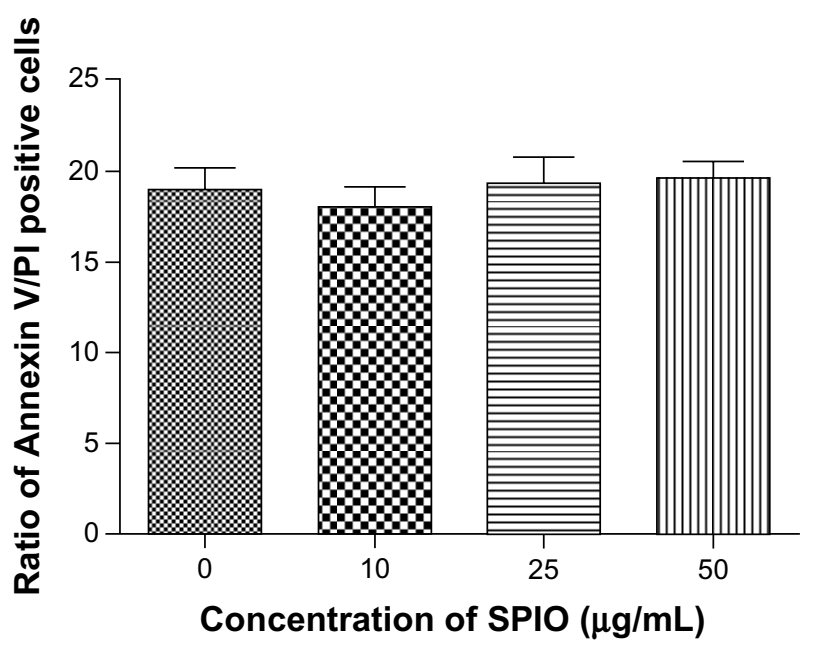

Figure 4 Cell apoptosis of DCs and SPIO-DCs determined by FCM at different labeling concentrations $(0,10,25$, and $50 \mu \mathrm{g} / \mathrm{mL} ; P=0.7747)$

Note: Ratio of Annexin V/PI positive cells refers to sum of ratio of Annexin V + PI + and Annexin $\mathrm{V}+\mathrm{PI}$-. Annexin $\mathrm{V}+\mathrm{PI}+$ demonstrated late apoptotic cells. Annexin $\mathrm{V}+\mathrm{Pl}$ - demonstrated early apoptotic cells.

Abbreviations: DCs, dendritic cells; FCM, flow cytometry; PI, propidium iodide; SPIO, superparamagnetic iron oxide.

inguinal lymph nodes 24 hours after the administration of DCs (Figure 6).

\section{EGFP signal localization in lymph nodes}

To determine whether SPIO labeling would influence DCs' migration in vivo, evidence of EGFP localization within the areas of draining lymph nodes was confirmed by fluorescence confocal microscopy. The positive EGFP fluorescence localized in the popliteal lymph nodes was observed and the fluorescence intensity and the number of cells decreased with increasing concentration of SPIO labeling. In contrast, no fluorescence was detected in inguinal lymph nodes during the first 24 hours (Figure 7).

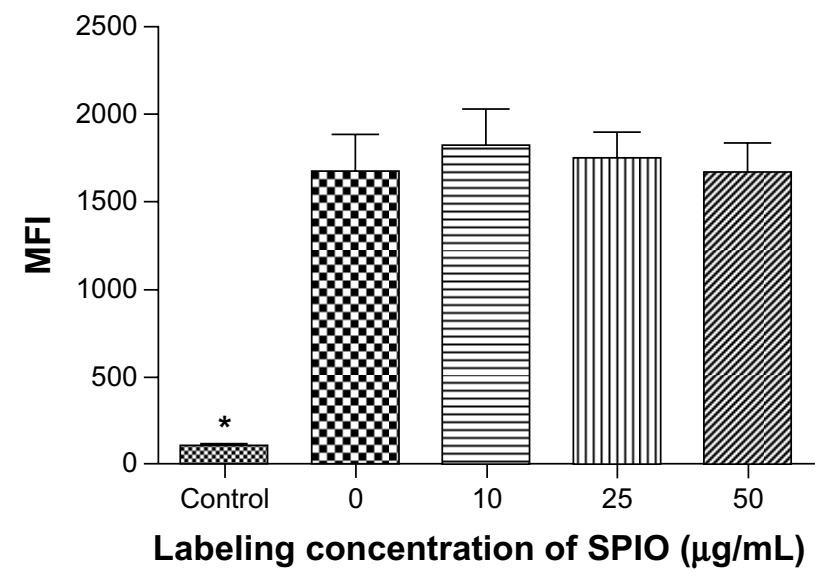

Figure $5 \mathrm{MFI}$ of EGFP-DCs after labeling with SPIO. Note: $* P=0.0017$.

Abbreviations: EGFP, enhanced green fluorescent protein; MFI, mean fluorescence intensity; SPIO, superparamagnetic iron oxide; DCs, dendritic cells.
After confirming differences in SPIO labeled EGFP positive DCs distribution in lymph nodes, the ratio of migrating DCs was examined by FCM. The number of DCs that migrated into the popliteal lymph nodes were $3.77 \%$, $3.03 \%, 2.27 \%$, and $1.23 \%$, respectively, according to SPIO labeling concentrations (Figure 7).

\section{Discussion}

Biotherapy for cancer has been of great interest to clinicians. However, many clinical trials designed with DCs to elicit immunity against solid tumors have not induced effective anti-tumor immunity. ${ }^{16,17}$ One of the reasons for the therapeutic deficiencies might be the fact that most DCs remain at the injection site and undergo apoptosis instead of migrating into lymphoid tissues. ${ }^{12,18}$ Since tracking DC migration in vivo is a novel non-invasive technique, the influence of different concentrations of the synthetic SPIO labeling on DCs was observed and, at the same time, the migration of SPIO labeled EGFP transgenic DCs was explored with an OPI approach in this study.

SPIO particles have been widely used as agents for cell labeling and tracking for magnetic resonance imaging. ${ }^{14,19}$ These nanoparticles have been confirmed to be delivered into DCs by our previous study. ${ }^{10}$ In this study, we examined DCs labeled with different concentrations of SPIO nanoparticles by Prussian blue staining and found that cellular iron increased gradually with the increase in ferric concentration (Figures 1 and 2). Changes in cell morphology were not observed under a light microscope, suggesting the morphology of DCs was minimally influenced by higher concentrations of SPIO labeling. Pauline Verdijk et $\mathrm{al}^{20}$ had shown an average of $30 \mu \mathrm{g}$ of intracellular iron per $1 \times 10^{6}$ cells without compromising cell viability by $200 \mu \mathrm{g}$ ferrumoxide/ $\mathrm{mL}$ labeling and 4 days co-culture time, which means this kind of synthetic SPIO used in the present study can be more easily inserted into DCs than other kinds of SPIO.

Before synthetic SPIO can be used to track DCs in vivo, the phenotypes of DCs following SPIO labeling should be considered. In this study, the expression of the major histocompatibility complex (MHC)-II molecule, the costimulatory molecules CD80, CD86, and the chemokine receptor CCR7 were investigated. These molecules were detected by FCM 12 hours after SPIO labeling and the negative results demonstrated that this type of synthetic SPIO had little effect on the phenotype of DCs. Besides these phenotypic effects, apoptosis of DCs with different intracellular iron contents were also assessed and no significant differences were found with respect to either early apoptosis or late 

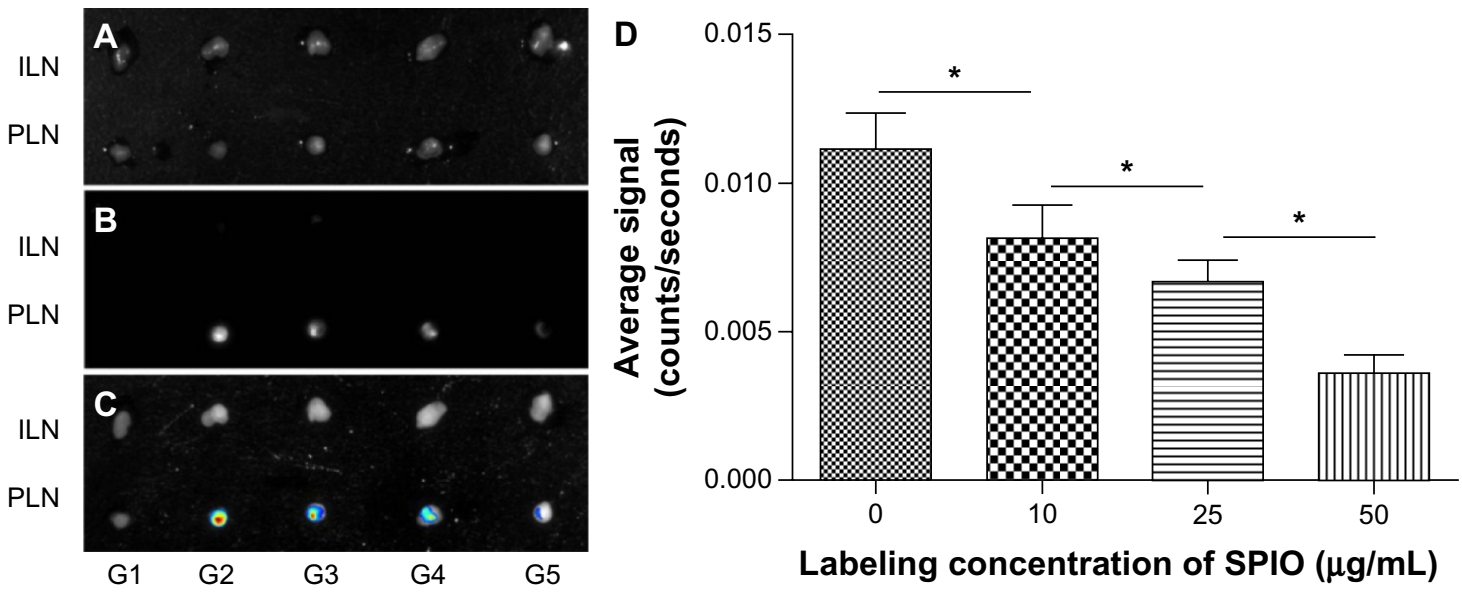

Figure 6 Ex vivo imaging of EGFP fluorescence signal in draining lymph nodes. (A) Overview of whole popliteal and inguinal lymph nodes; (B) draining lymph node imaging of EGFP signals ex vivo; (C) fluorescence signals analyzed by image software; (D) the measured average signal to background.

Note: $* P=0.0273<0.05$

Abbreviations: DCs, dendritic cells; EGFP, enhanced green fluorescent protein; GI, phosphate buffered saline; G2, DCs; G3, DCs labeled with SPIO (I0 $\mu$ g/mL); G4, DCs labeled with SPIO (25 $\mu \mathrm{g} / \mathrm{mL})$; G5, DCs labeled with SPIO (50 $\mathrm{g} / \mathrm{mL})$; ILN, inguinal lymph nodes; PLN, popliteal lymph nodes; SPIO, superparamagnetic iron oxide.
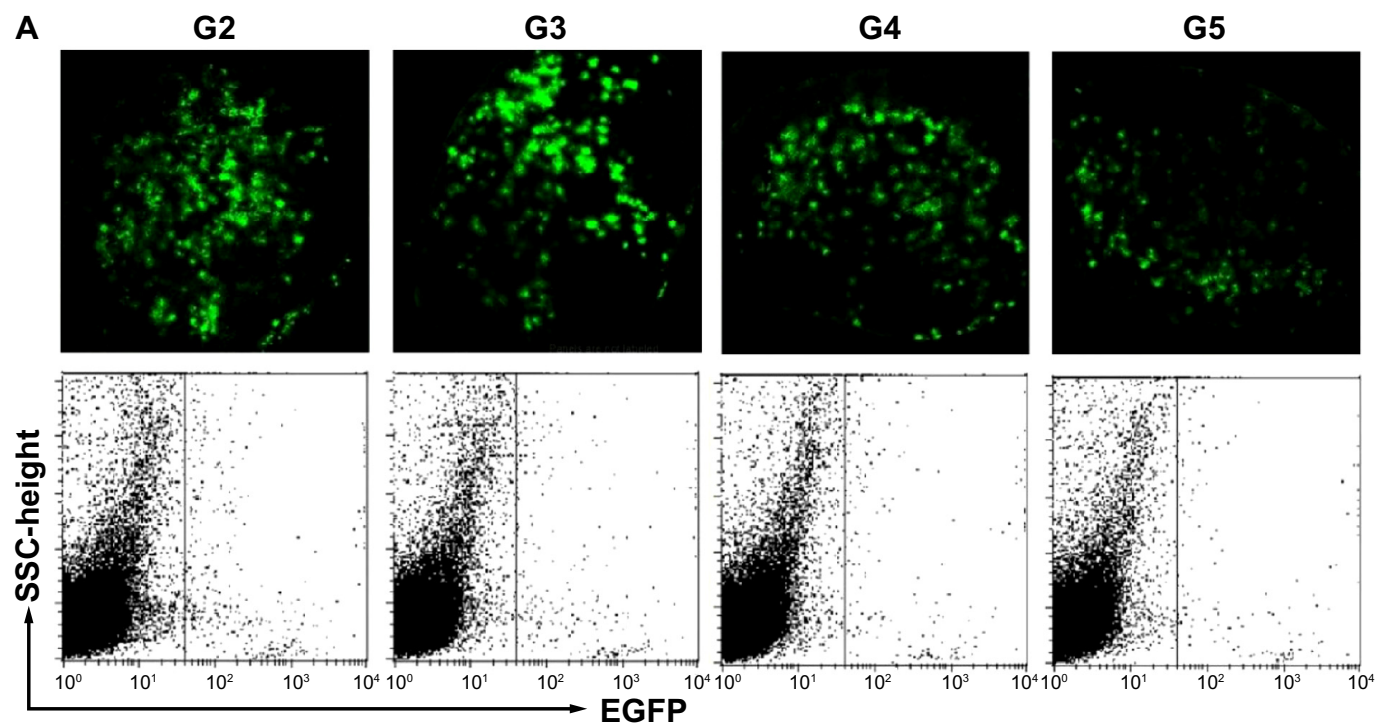

B

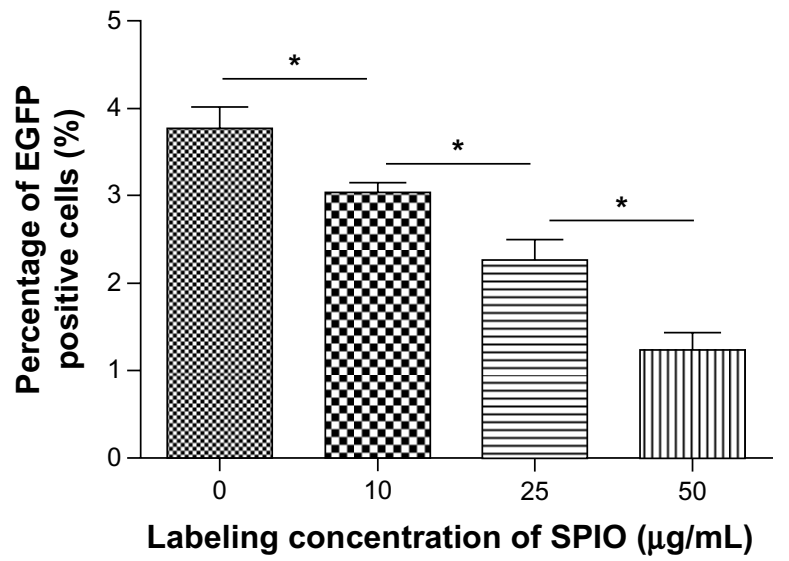

Figure 7 Accumulation of DCs in the draining lymph nodes $(\times 400)$. (A) Analysis of EGFP fluorescence by FCM; (B) quantitative analyses of EGFP fluorescence in popliteal LNs. Note: $* P=0.0156$.

Abbreviations: EGFP, enhanced green fluorescent protein; FCM, flow cytometry; G2, DCs; G3, DCs labeled with SPIO (I0 $\mu \mathrm{g} / \mathrm{mL})$; G4, DCs labeled with SPIO (25 $\mu \mathrm{g} / \mathrm{mL})$; G5, DCs labeled with SPIO (50 $\mathrm{g} / \mathrm{mL})$; SPIO, superparamagnetic iron oxide; DCs, dendritic cells; SSC, side scatter; LN, lymph node. 
apoptosis (Figures 3 and 4). From the data mentioned above, it is evident that synthetic SPIO labeling did not interfere with DCs' maturation, expression of migration molecules, or apoptosis, which was similar to other kinds of SPIO nanoparticles modified by different materials. ${ }^{21,22}$

To analyze the migratory efficiency of DCs loaded with different concentrations of SPIO in vivo, the OPI technique was adopted. Because dying cells would lose their autofluorescence rapidly, EGFP were not only regarded as a tool for tracking cell migration, but also a marker for the viability of cells. ${ }^{23}$ Based on EGFPs' continuous expression compared to other fluorescent dyes, DCs were induced from bone marrow monocytes of EGFP transgenic mice. An important aspect of the migration ability of EGFP-DCs loaded with SPIO is whether the fluorescence intensity of EGFP-DCs would be influenced by SPIO labeling. The FCM results showed that the intensity of the fluorescent signal of EGFP in DCs did not weaken with different concentrations of SPIO labeling compared to EGFP-DCs alone, which means that fluorescence intensity of EGFP in DCs were minimally influenced by synthetic SPIO endocytosis (Figure 5).

Efficacy of biotherapies based on DCs requires a sufficient number of DCs to migrate into appropriate tissues to induce or modulate immune responses and present relevant antigens to naïve $\mathrm{T}$ cells in draining lymph nodes of infection or tumor. Although migration efficiency was ineffective, we demonstrated that SPIO-EGFP-DCs could migrate into draining popliteal lymph nodes though footpad injection with a specific SPIO labeling concentration of $25 \mu \mathrm{g} / \mathrm{mL} .{ }^{11}$ To define the homing capabilities of DCs labeled with different concentrations of synthetic SPIO, OPI was performed in this study, which has recently gained attention for tracking cells labeled with fluorescent dyes in vivo because of its high spatial resolution. Our results showed strong EGFP expression in popliteal lymph nodes during the first 24 hours and the intensity of fluorescence weakened gradually with the increase in labeling concentrations of SPIO (Figure 6). The optical images also verified that the fluorescence signals of EGFP existed within popliteal lymph nodes in the first 24 hours. On the contrary, it was not detected in the secondary draining inguinal lymph nodes, which was also in accordance with other studies. ${ }^{24-26}$ Further similar evidence of EGFP expression detected by immunohistochemistry and FCM was revealed in which DCs without SPIO labeling had the strongest migration ability and those with the most content of intracellular SPIO had the weakest migration ability during the first 24 hours after DCs' infusion (Figure 7).
Based on the data presented here, there was sufficient proof to confirm the migration of EGFP-DCs with different concentrations of synthetic SPIO labeling into the first draining lymph nodes in the short term, which suggests that DCs would maintain stronger migration capability when labeled with a low dose of SPIO when tracking them in vivo. Consequently, further experiments exploring the mechanisms behind DC migratory capabilities with different concentrations of the synthetic SPIO are currently underway.

\section{Acknowledgments}

We acknowledge the kind support of Prof Ning Gu, Yayi Hou, and Zichun Hua for technical assistance. The authors are grateful for grants from the National Important Science Research Program of China (2011CB933503), the National Natural Sciences Foundation of China (81271698), the Project of Natural Science Foundation of Jiangsu Province (BK2012744, BK2012075), and the Nanjing Medical Development Foundation (ZKX10031, ZKX12034, 201201077).

\section{Disclosure}

The authors report no conflicts of interest in this work.

\section{References}

1. Grazú V, Silber AM, Moros M, et al. Application of magnetically induced hyperthermia in the model protozoan Crithidia fasciculata as a potential therapy against parasitic infections. Int $J$ Nanomedicine. 2012;7:5351-5360.

2. Raoof M, Corr SJ, Kaluarachchi WD, et al. Stability of antibodyconjugated gold nanoparticles in the endolysosomal nanoenvironment: implications for noninvasive radiofrequency-based cancer therapy. Nanomedicine. 2012;8(7):1096-1105.

3. Chen R, Yu H, Jia ZY, Yao QL, Teng GJ. Efficient nano iron particlelabeling and noninvasive MR imaging of mouse bone marrow-derived endothelial progenitor cells. Int J Nanomedicine. 2011;6:511-519.

4. Daldrup-Link HE, Rudelius M, Piontek G, et al. Migration of iron oxide-labeled human hematopoietic progenitor cells in a mouse model: in vivo monitoring with $1.5-\mathrm{T}$ MR imaging equipment. Radiology. 2005;234(1):197-205.

5. Murase K, Oonoki J, Takata H, et al. Simulation and experimental studies on magnetic hyperthermia with use of superparamagnetic iron oxide nanoparticles. Radiol Phys Technol. 2011;4(2):194-202.

6. Mori Y, Umeda M, Fukunaga M, Ogasawara K, Yoshioka Y. MR contrast in mouse lymph nodes with subcutaneous administration of iron oxide particles: size dependency. Magn Reson Med Sci. 2011;10(4):219-227.

7. McBain SC, Yiu HH, Dobson J. Magnetic nanoparticles for gene and drug delivery. Int J Nanomedicine. 2008;3(2):169-180.

8. O'Neill DW, Adams S, Bhardwaj N. Manipulating dendritic cell biology for the active immunotherapy of cancer. Blood. 2004;104(8):2235-2246.

9. Xu Q, Liu G, Yuan X, et al. Antigen-specific T-cell response from dendritic cell vaccination using cancer stem-like cell-associated antigens. Stem Cells. 2009;27(8):1734-1740.

10. Mou Y, Chen B, Zhang Y, et al. Influence of synthetic superparamagnetic iron oxide on dendritic cells. Int J Nanomedicine. 2011;6:1779-1786. 
11. Mou Y, Hou Y, Chen B, et al. In vivo migration of dendritic cells labeled with synthetic superparamagnetic iron oxide. Int $J$ Nanomedicine. 2011;6:2633-2640.

12. Verdijk P, Aarntzen EH, Lesterhuis WJ, et al. Limited amounts of dendritic cells migrate into the T-cell area of lymph nodes but have high immune activating potential in melanoma patients. Clin Cancer Res. 2009;15(7):2531-2540.

13. Xie H, Hua C, Sun L, et al. 17ß-estradiol induces CD40 expression in dendritic cells via MAPK signaling pathways in a minichromosome maintenance protein 6-dependent manner. Arthritis Rheum. 2011;63(8):2425-2435.

14. Yao K, Yun J, Shen S, Wang L, He X, Yu X. Characterization of a novel continuous supermacroporous monolithic cryogel embedded with nanoparticles for protein chromatography. $J$ Chromatogr A. 2006;1109(1):103-110.

15. Ge Y, Zhang Y, Xia J, et al. Effect of surface charge and agglomerate degree of magnetic iron oxide nanoparticles on KB cellular uptake in vitro. Colloids Surf B Biointerfaces. 2009;73(2):294-301.

16. Norian LA, Rodriguez PC, O’Mara LA, et al. Tumor-infiltrating regulatory dendritic cells inhibit CD8+ T cell function via L-arginine metabolism. Cancer Res. 2009;69(7):3086-3094.

17. Liu Q, Zhang C, Sun A, Zheng Y, Wang L, Cao X. Tumor-educated CD11bhighIalow regulatory dendritic cells suppress $\mathrm{T}$ cell response through arginase I. J Immunol. 2009;182(10):6207-6216.

18. De Vries IJ, Krooshoop DJ, Scharenborg NM, et al. Effective migration of antigen-pulsed dendritic cells to lymph nodes in melanoma patients is determined by their maturation state. Cancer Res. 2003;63(1):12-17.
19. Yao Y, Wang Y, Zhang Y, et al. In vivo imaging of macrophages during the early-stages of abdominal aortic aneurysm using high resolution MRI in ApoE mice. PLoS ONE. 2012;7(3):e33523.

20. Verdijk P, Scheenen TW, Lesterhuis WJ, et al. Sensitivity of magnetic resonance imaging of dendritic cells for in vivo tracking of cellular cancer vaccines. Int J Cancer. 2007;120(5):978-984.

21. Guo L, Huang J, Zheng LM. Control generating of bacterial magnetic nanoparticle-doxorubicin conjugates by poly-L-glutamic acid surface modification. Nanotechnology. 2011;22(17):175102.

22. Sun Z, Yathindranath V, Worden M, et al. Characterization of cellular uptake and toxicity of aminosilane-coated iron oxide nanoparticles with different charges in central nervous system-relevant cell culture models. Int J Nanomedicine. 2013;8:961-970.

23. Eggert AA, van der Voort R, Torensma R, et al. Analysis of dendritic cell trafficking using EGFP-transgenic mice. Immunol Lett. 2003;89(1):17-24.

24. MartIn-Fontecha A, Sebastiani S, Höpken UE, et al. Regulation of dendritic cell migration to the draining lymph node: impact on T lymphocyte traffic and priming. J Exp Med. 2003;198(4):615-621.

25. Pham W, Xie J, Gore JC. Tracking the migration of dendritic cells by in vivo optical imaging. Neoplasia. 2007;9(12):1130-1137.

26. Christian NA, Benencia F, Milone MC, et al. In vivo dendritic cell tracking using fluorescence lifetime imaging and near-infrared-emissive polymersomes. Mol Imaging Biol. 2009;11(3):167-177.
International Journal of Nanomedicine

\section{Publish your work in this journal}

The International Journal of Nanomedicine is an international, peerreviewed journal focusing on the application of nanotechnology in diagnostics, therapeutics, and drug delivery systems throughout the biomedical field. This journal is indexed on PubMed Central, MedLine, CAS, SciSearch $\AA$, Current Contents ${ }^{\circledR} /$ Clinical Medicine,

\section{Dovepress}

Journal Citation Reports/Science Edition, EMBase, Scopus and the Elsevier Bibliographic databases. The manuscript management system is completely online and includes a very quick and fair peer-review system, which is all easy to use. Visit http://www.dovepress.com/ testimonials.php to read real quotes from published authors. 